\title{
A characterization of panconnected graphs satisfying a local ore-type condition
}

Armen S. Asratian, R. Häggkvist and G. V. Sarkisian

The self-archived postprint version of this journal article is available at Linköping University Institutional Repository (DiVA):

http:/ / urn.kb.se/ resolve?urn=urn:nbn:se:liu:diva- 143770

N.B.: When citing this work, cite the original publication.

Asratian, A. S., Häggkvist, R., Sarkisian, G. V., (1996), A characterization of panconnected graphs satisfying a local ore-type condition, J ournal of Graph Theory, 22(2), 95-103.

https:/ / doi.org/ 10.1002/(SICI)1097-0118(199606)22:2\&tt;95::AID-J GT1\&gt;3.0.CO;2-F

Original publication available at:

https:/ / doi.org/ 10.1002/ (SICI)1097-0118(199606)22:2\&lt;95: :AID-

JGT1\&gt;3.0.CO;2-F

Copyright: Wiley (12 months)

http:// eu.wiley.com/WileyCDA/ 


\section{A Characterization of Panconnected Graphs Satisfying a Local Ore-Type Condition}

A. S. Asratian

DEPARTMENT OF MATHEMATICS UNIVERSITY OF UMEA S-901 87, UMEÅ, SWEDEN DEPARTMENT OF MATHEMATICAL

CYBERNETICS

YEREVAN STATE UNIVERSITY

YEREVAN, 375049, REPUBLIC OF ARMENIA

R. Häggkvist

DEPARTMENT OF MATHEMATICS

UNIVERSITY OF UMEA S-901 87, UMEÅ, SWEDEN

G. V. Sarkisian DEPARTMENT OF MATHEMATICAL CYBERNETICS YEREVAN STATE UNIVERSITY YEREVAN, 375049, REPUBLIC OF ARMENIA

\section{ABSTRACT}

It is well known that a graph $G$ of order $p \geq 3$ is Hamilton-connected if $d(u)+d(v) \geq p+1$ for each pair of nonadjacent vertices $u$ and $v$. In this paper we consider connected graphs $G$ of order at least 3 for which $d(u)+d(v) \geq|N(u) \cup N(v) \cup N(w)|+1$ for any path $u w v$ with $u v \notin E(G)$, where $N(x)$ denote the neighborhood of a vertex $x$. We prove that a graph $G$ satisfying this condition has the following properties: (a) For each pair of nonadjacent vertices $x, y$ of $G$ and for each integer $k, d(x, y) \leq k \leq|V(G)|-1$, there is an $x-y$ path of length $k$. (b) For each edge $x y$ of $G$ and for each integer $k$ lexcepting maybe one $k \in\{3,4\}$ ) there is a cycle of length $k$ containing $x y$.

Consequently $G$ is panconnected (and also edge pancyclic) if and only if each edge of 
$G$ belongs to a triangle and a quadrangle.

Our results imply some results of Williamson, Faudree, and Schelp. (C) 1996 John Wiley \& Sons, inc.

\section{INTRODUCTION}

We use Bondy and Murty [6] for terminology and notation not defined here and consider finite simple graphs only. For each vertex $u$ of a graph $G$ we denote by $N(u)$ the set of all vertices of $G$ adjacent to $u$. The distance between vertices $u$ and $v$ is denoted by $d(u, v)$. A path with $x$ and $y$ as end vertices is called an $x-y$ path. A path is called a Hamilton path if it contains all the vertices of $G$. A graph $G$ is Hamilton-connected if every two vertices of $G$ are connected by a Hamilton path.

Let $G$ be a graph of order $p \geq 3$. $G$ is called panconnected if for each pair of distinct vertices $x$ and $y$ of $G$ and for each $l, d(x, y) \leq l \leq p-1$, there is an $x-y$ path of length $l$. $G$ is called pancyclic if it contains a cycle of length $l$ for each $l$ satisfying $3 \leq l \leq p$. $G$ is called a vertex pancyclic (edge pancyclic) if each vertex (edge) of $G$ lies on a cycle of every length from 3 to $p$ inclusive.

The following results are known.

Theorem 1. (Ore [12]). Let $G$ be a graph of order $p \geq 3$, where $d(u)+d(v) \geq p+1$ for each pair $u, v$ of nonadjacent vertices. Then $G$ is Hamilton-connected.

Theorem 2. (Williamson [13]). A connected graph of order $p \geq 3$ is panconnected if any of the following two conditions hold:

(a) $d(u) \geq(p+2) / 2$ for each vertex $u$ of $G$,

(b) $d(u)+d(v) \geq(3 p-2) / 2$ for each pair of nonadjacent vertices $u, v$ of $G$.

Theorem 3. (Faudree and Schelp [8]). If $G$ is a graph of order $p \geq 5$ with $d(u)+d(v) \geq$ $p+1$ for each pair of nonadjacent vertices $u, v$ then $G$ contains a path of every length from 4 to $n-1$ inclusive, between any pair of distinct vertices of $G$.

A shorter proof of Theorem 3 was given by Cai [7]. From results of Bondy [5] and Häggkvist et al. [10] it follows that every graph $G$ satisfying the condition of Theorem 1 is pancyclic. Some other properties of graphs satisfying the condition of Theorem 1 were obtained in $[4,9,14,15]$.

The following generalization of Theorem 1 was found by Asratian et al. [1].

Theorem 4. [1]. Let $G$ be a connected graph of order at least 3 where $d(u)+d(v) \geq$ $|N(u) \cup N(v) \cup N(w)|+1$ for any path $u w v$ with $u v \notin E(G)$. Then $G$ is Hamilton-connected.

Denote by $L$ the set of all graphs satisfying the condition of Theorem 4 . It was proved in [3] that every graph from $L$ is pancyclic, and in [2] it was shown that a graph $G \in L$ is vertex pancyclic if and only if each vertex of $G$ lies on a triangle.

In this paper we show that a graph $G \in L$ has the following properties:

(a) For each pair of nonadjacent vertices $x, y$ of $G$ and for each integer $n, d(x, y) \leq n \leq$ $|V(G)|-1$, there is an $x-y$ path of length $n$. 
(b) For each edge $x y$ and for each integer $k, 3 \leq n \leq|V(G)|$, (excepting maybe one $k \in\{3,4\}$ ) there is a cycle of length $k$ containing $x y$.

This implies that a graph $G \in L$ is panconnected (and also edge pancyclic) if and only if each edge of $G$ lies on a triangle and on a quadrangle.

Note that for each $r \geq 2$ and each $p \geq 3$ there exists a panconnected graph $G_{r, p} \in L$ of order $p r$ with diameter $r$ : its vertex set is $\cup_{i=0}^{r} V_{i}$ where $V_{0}, V_{1}, \ldots, V_{r}$ are pairwise disjoint sets of cardinality $p$ and two vertices are adjacent if and only if they both belong to $V_{i} \cup V_{i+1}$ for some $i \in\{0,1, \ldots, r-1\}$.

\section{NOTATION AND PRELIMINARY RESULTS}

Let $P$ be a path of $G$. We denote by $\vec{P}$ the path $P$ with a given orientation and by $\overleftarrow{P}$ the path $P$ with the reverse orientation. If $u, v \in V(P)$, then $u \vec{P} v$ denotes the consecutive vertices of $P$ from $u$ to $v$ in the direction specified by $\vec{P}$. The same vertices, in reverse order, are given by $v \bar{P} u$. We use $w^{+}$to denote the successor of $w$ on $\vec{P}$ and $w^{-}$to denote its predecessor. We denote by $N(P)$ the set of vertices $v$ outside $P$ with $N(v) \cap V(P) \neq \emptyset$. If $W \subseteq V(P)$ then $W^{+}=\left\{w^{+} / w \in W\right\}$ and $W^{-}=\left\{w^{-} / w \in W\right\}$.

We will say that a path $\vec{P}$ contains a triangle $a_{1} a_{2} a_{3} a_{1}$ if $a_{1}, a_{2}, a_{3} \in V(P), a_{1} a_{3} \in E(G)$ and $a_{1}^{+}=a_{2}=a_{3}^{-}$. A path $\vec{P}$ containing a triangle $\Delta$ is denoted by $\vec{P}^{\Delta}$. The set of all triangles contained in $\vec{P}^{\Delta}$ we denote by $T\left(\vec{P}^{\Delta}\right)$. We assume that an $x-y$ path $\vec{P}$ has an orientation from $x$ to $y$. A path on $n$ vertices will be denoted by $P_{n}$.

Let $A$ and $B$ be two disjoint subsets of vertices of a graph $G$. We denote by $\varepsilon(A, B)$ the number of edges in $G$ with one end in $A$ and the other in $B$.

Proposition 1. [11]. $G \in L$ if and only if for any path $u w v$ with $u v \notin E(G) \mid N(u) \cap$ $N(v)|\geq| N(w) \backslash(N(u) \cup N(v)) \mid+1$ holds.

Corollary 1. If $G \in L$ then $G$ is 3-connected and $|N(u) \cap N(v)| \geq 3$ for each pair of vertices $u, v$ with $d(u, v)=2$.

Proof. Let $d(u, v)=2$. If $w \in N(u) \cap N(v)$ then $u, v \in N(w) \backslash(N(u) \cup N(v))$ and, by Proposition 1, $|N(u) \cap N(v)| \geq 3$. This implies that $G$ is 3-connected.

Proposition 2. Let $G \in L$ and $x, y$ be two vertices of $G$ with $d(x, y)=l \geq 2$. Then there exists an $x-y$ path $P_{l+2}^{\Delta}$.

Proof. Let $P=u_{0} u_{1} \cdots u_{l}$ be an $x-y$ path of length $l=d(x, y)$ where $u_{0}=x$ and $u_{l}=y$. If there is a vertex outside $P$ which is adjacent to two consecutive vertices of $P$ then there is an $x-y$ path $P_{l+2}^{\Delta}$. Suppose that there is no such vertex outside $P$. Since $d\left(u_{0}, u_{2}\right)=$ 2 then, by Proposition 1, we have $\left|N\left(u_{0}\right) \cap N\left(u_{2}\right)\right| \geq\left|N\left(u_{1}\right) \backslash\left(N\left(u_{0}\right) \cup\left(u_{2}\right)\right)\right|+1 \geq 3$. Clearly,

$$
N\left(u_{0}\right) \cap V(P)=N\left(u_{0}\right) \cap N\left(u_{2}\right) \cap V(P)=\left\{u_{1}\right\} .
$$

Let $N\left(u_{0}\right) \cap N\left(u_{2}\right)=\left\{w_{1}, \ldots, w_{k}\right\}$ where $k \geq 3$ and $w_{1}=u_{1}$. Furthermore, let $\mid N\left(w_{1}\right) \cap$ $N\left(w_{2}\right) \mid=m$. If $w_{i} w_{j} \notin E(G)$ for each pair $i, j, 1 \leq i<j \leq k$, then using (1) and 
Proposition 1 we obtain

$$
m=\left|N\left(w_{1}\right) \cap N\left(w_{2}\right)\right| \geq 1+\left|N\left(u_{0}\right) \backslash\left(N\left(w_{1}\right) \cup N\left(w_{2}\right)\right)\right| \geq k+1 .
$$

Furthermore, since $N\left(w_{1}\right) \cap N\left(w_{2}\right) \subseteq N\left(w_{1}\right)=N\left(w_{1}\right) \backslash\left(N\left(u_{0}\right) \cup N\left(u_{2}\right)\right)$ then $k=$ $\left|N\left(u_{0}\right) \cap N\left(u_{2}\right)\right| \geq 1+\left|N\left(w_{1}\right) \backslash\left(N\left(u_{0}\right) \cup N\left(u_{2}\right)\right)\right| \geq 1+m$, which contradicts (2). Hence $w_{i} w_{j} \in E(G)$ for some pair $i, j$. Then there is an $x-y$ path $P_{l+2}^{\Delta}=u_{0} w_{i} w_{j} u_{2} \cdots u_{l}$ with $\Delta=x w_{i} w_{j} x$.

Proposition 3. Let $G \in L$ and $x y \in E(G)$. Then there exists an $x-y$ path $P_{n}^{\Delta}$ where $4 \leq n \leq 6$.

Proof. Two cases are possible.

Case 1. $x y$ does not lie on a triangle.

Since $G$ is 3-connected we have $d(x) \geq 3$. Let $u_{1} x \in E(G)$ and $u_{1} \neq y$. Since $d\left(u_{1}, y\right)=$ 2 and $\left|N(y) \cap N\left(u_{1}\right)\right| \geq 2$ there exists a vertex $u_{2} \in N\left(u_{1}\right) \cap N(y), u_{2} \neq x$. Consider a path $P=u_{0} u_{1} u_{2} u_{3}$ where $u_{0}=x$ and $u_{3}=y$. Clearly, $u_{0} u_{2}, u_{1} u_{3} \notin E(G), d\left(u_{0}, u_{2}\right)=2$ and $u_{0} u_{3} \in E(G)$. Now we can prove, by repeating the proof of Proposition 2 with (1) changed to $N\left(u_{0}\right) \cap V(P)=N\left(u_{0}\right) \cap N\left(u_{2}\right) \cap V(P)=\left\{u_{1}, u_{3}\right\}$, that there exists an $u_{0}-u_{3}$ path $P_{5}^{\Delta}$. Consequently there exists an $x-y$ path $P_{5}^{\Delta}$, because $x=u_{0}$ and $y=u_{3}$.

Case 2. $x y$ lies on a triangle $x y z x$.

Since $G$ is 3 -connected we have $d(z) \geq 3$. If there is a vertex $u \in N(z) \backslash\{x, y\}$ such that $u x \in E(G)$ or $u y \in E(G)$ then we have an $x-y$ path $P_{4}^{\Delta}$.

If no such vertex exists then $u x, u y \notin E(G)$ for each vertex $u \in N(z) \backslash\{x, y\}$. Consider a vertex $w \in N(z) \backslash\{x, y\}$. Then $d(w, x)=2$ and there is a vertex $u_{1} \in(N(x) \cap N(w)) \backslash\{z\}$. Consider a path $P=u_{0} u_{1} u_{2} u_{3}$ where $u_{0}=x, u_{2}=w, u_{3}=z$. Clearly, $y u_{3} \in E(G)$ and $y u_{1}, y u_{2}, u_{0} u_{2}, u_{1} u_{3} \notin E(G)$. Using the same arguments as in Case 1 we will obtain that there is an $u_{0}-u_{3}$ path $P_{5}^{\Delta}$. Since $x=u_{0}$ and $y u_{3} \in E(G)$ then there is an $x-y$ path $P_{6}^{\Delta}$.

\section{MAIN RESULTS}

Theorem 5. Let $G \in L$ and $x, y$ be two distinct vertices of $G$. If there exists an $x-y$ path $P_{n}^{\Delta}$ such that $4 \leq n \leq|V(G)|-2$ then there exists an $x-y$ path $P_{n+t}^{\Delta_{1}}$ where $1 \leq t \leq 2$.

Proof. Since $G$ is connected and $n<|V(G)|$ then $N\left(P_{n}^{\Delta}\right) \neq \emptyset$. For each $v \in N\left(P_{n}^{\Delta}\right)$ we denote by $W_{v}$ the set $N(v) \cap V\left(P_{n}^{\Delta}\right)$. Let $U_{1}=\left\{v \in N\left(P_{n}^{\Delta}\right) /\left|W_{v}\right|=1\right\}$ and $U_{2}=\{v \in$ $N\left(P_{n}^{\Delta}\right) /\left|W_{v}\right| \geq 2$ and $\left.W_{v} \backslash\{x, y\} \neq \emptyset\right\}$.

Suppose there does not exist an $x-y$ path $P_{n+t}^{\Delta_{1}}$, where $1 \leq t \leq 2$. Then the following properties hold.

Property 1. $v w^{+} \notin E(G)$ for each $v \in N\left(P_{n}^{\Delta}\right)$ and each $w \in W_{v} \backslash\{y\}$.

Property 2. If $v \in U_{1}, W_{v}=\{w\}$ and $w \notin\{x, y\}$ then the set $T\left(P_{n}^{\Delta}\right)$ contains the unique triangle $w^{-} w w^{+} w^{-}$.

Proof. Let $a_{1} a_{2} a_{3} a_{1}$ be a triangle from the set $T\left(P_{n}^{\Delta}\right)$. Suppose $a_{2} \neq w$. Since $d\left(v, w^{-}\right)=$ $2=d\left(v, w^{+}\right)$then, by Corollary 1 , there exist vertices $v_{1}$ and $v_{2}$ such that $v_{1} \in(N(v) \cap$ 
$\left.N\left(w^{-}\right)\right) \backslash V\left(P_{n}^{\Delta}\right)$ and $v_{2} \in\left(N(v) \cap N\left(w^{+}\right)\right) \backslash V\left(P_{n}^{\Delta}\right)$. This gives an $x-y$ path

$$
P_{n+2}^{\Delta_{1}}= \begin{cases}x \vec{P}_{n}^{\Delta} w^{-} v_{1} v w \vec{P}_{n}^{\Delta} y & \text { if } a_{2} \in w^{+} \vec{P}_{n}^{\Delta} y \\ x \vec{P}_{n}^{\Delta} w v v_{2} w^{+} \vec{P}_{n}^{\Delta} y & \text { if } a_{2} \in x \vec{P}_{n}^{\Delta} w^{-}\end{cases}
$$

with $\Delta_{1}=a_{1} a_{2} a_{3} a_{1}$ such that $V\left(P_{n}^{\Delta}\right) \subset V\left(P_{n+2}^{\Delta_{1}}\right)$, a contradiction.

Property 3. $U_{2} \neq \emptyset$.

Proof. Since $G$ is 3-connected then there exists a vertex $v \in N\left(P_{n}^{\Delta}\right)$ such that $W_{v} \backslash$ $\{x, y\} \neq \emptyset$. Let $w \in W_{v} \backslash\{x, y\}$. If $v \notin U_{2}$ then $v \in U_{1}$ and, by Property $2, w^{-} w w^{+} w^{-}$is the unique triangle in the set $T\left(P_{n}^{\Delta}\right)$. Since $d\left(v, w^{+}\right)=2,\left|W_{v}\right|=1$ and $\left|N(v) \cap N\left(w^{+}\right)\right| \geq 3$ then there is a vertex $u \in\left(N(v) \cap N\left(w^{+}\right)\right) \backslash V\left(P_{n}^{\Delta}\right)$. By Property 2, $u \notin U_{1}$. Therefore $u \in U_{2}$.

Property 4. Let $v \in U_{2}$ and $Q$ be a subset of the set $W_{v}=\left\{w_{1}, \ldots, w_{p}\right\}$ such that $y \notin Q$. Then

$$
\sum_{w_{i} \in Q}\left|N(v) \cap N\left(w_{i}^{+}\right)\right| \geq \sum_{w_{i} \in Q}\left(\left|N\left(w_{i}\right) \backslash\left(N(v) \cup N\left(w_{i}^{+}\right)\right)\right|+1\right)
$$

Furthermore, if $a_{1} a_{2} a_{3} a_{1}$ is a triangle from the set $T\left(P_{n}^{\Delta}\right)$ with $\left\{a_{1}, a_{2}\right\} \cap Q=\emptyset$ then

$$
N(v) \cap N\left(w_{i}^{+}\right) \subseteq W_{v} \quad \text { for each } w_{i} \in Q
$$

and

$$
w_{i}^{+} w_{j}^{+} \notin E(G) \text { for each pair of vertices } w_{i}, w_{j} \in Q
$$

Proof. Clearly, (3) follows from Proposition 1. If (4) does not hold for some $w_{i} \in Q$ then there is a vertex $v_{1} \in\left(N(v) \cap N\left(w_{i}^{+}\right)\right) \backslash W_{v}$ and an $x-y$ path $P_{n+2}^{\Delta_{1}}=x \vec{P}_{n}^{\Delta} w_{i} v v_{1} w_{i}^{+} \vec{P}_{n}^{\Delta} y$ with $\Delta_{1}=a_{1} a_{2} a_{3} a_{1}$, a contradiction. So (4) holds. If (5) does not hold then $w_{i}^{+} w_{j}^{+} \in$ $E(G)$ for some pair of vertices $w_{i}, w_{j} \in Q$ where $i<j$. Then there is an $x-y$ path $P_{n+1}^{\Delta_{1}}=x \vec{P}_{n}^{\Delta} w_{i} v w_{j} \overleftarrow{P}_{n}^{\Delta} w_{i}^{+} w_{j}^{+} \vec{P}_{n}^{\Delta} y$ with

$$
\Delta_{1}= \begin{cases}a_{1} a_{2} a_{3} a_{1} & \text { if } a_{1} \notin w_{i}^{+} \vec{P}_{n}^{\Delta} w_{j} \\ a_{3} a_{2} a_{1} a_{3} & \text { otherwise. }\end{cases}
$$

a contradiction. So (5) holds.

Property 5. Let $a_{1} a_{2} a_{3} a_{1}$ be a triangle from the set $T\left(P_{n}^{\Delta}\right)$. Then $\left\{a_{1}, a_{2}\right\} \cap W_{v} \neq \emptyset \neq$ $\left\{a_{2}, a_{3}\right\} \cap W_{v}$ for each vertex $v \in U_{2}$.

Proof. Suppose that $\left\{a_{1}, a_{2}\right\} \cap W_{v}=\emptyset$ and let $w_{1}, \ldots, w_{p}$ denote the vertices of $W_{v}$ occurring on $P_{n}^{\Delta}$ in the order of their indices. Set $Q=\left\{w_{1}, \ldots, w_{p-1}\right\}$. Then, by Property 4 , we have (3), (4), and (5). Since $w_{p}$ can be adjacent to each vertex $w_{i}^{+}$then

$$
\sum_{w_{i} \in Q}\left|N(v) \cap N\left(w_{i}^{+}\right)\right| \leq \varepsilon\left(Q, Q^{+}\right)+p-1 .
$$


Furthermore,

$$
\sum_{w_{i} \in Q}\left|N\left(w_{i}\right) \backslash\left(N(v) \cup N\left(w_{i}^{+}\right)\right)\right| \geq \varepsilon\left(Q, Q^{+}\right)+p-1
$$

since $v \notin Q^{+}$and $v \in N\left(w_{i}\right) \backslash\left(N(v) \cup N\left(w_{i}^{+}\right)\right)$for each $i=1, \ldots, p-1$. Clearly, (7) is equivalent to

$$
\sum_{w_{i} \in Q}\left(\left|N\left(w_{i}\right) \backslash\left(N(v) \cup N\left(w_{i}^{+}\right)\right)\right|+1\right) \geq \varepsilon\left(Q, Q^{+}\right)+2(p-1) .
$$

But (6) and (8) contradict (3). So $\left\{a_{1}, a_{2}\right\} \cap W_{v} \neq \emptyset$.

We can prove $\left\{a_{3}, a_{2}\right\} \cap W_{v} \neq \emptyset$ by considering the path $\bar{P}_{n}^{\Delta}$ and the triangle $a_{3} a_{2} a_{1} a_{3}$ and using the above arguments.

Property 6. $\left|W_{v}\right| \geq 3$ for each vertex $v \in U_{2}$.

Proof. Let $\Delta=a_{1} a_{2} a_{3} a_{1}$ be a triangle from the set $T\left(P_{n}^{\Delta}\right)$. Suppose $W_{v}=\left\{w_{1}, w_{2}\right\}$ for some $v \in U_{2}$ where $w_{1}$ and $w_{2}$ occur on $P_{n}^{\Delta}$ in the order of their indices. Since $v \in U_{2}$ then $W_{v} \backslash\{x, y\} \neq \emptyset$. W.l.o.g. we assume $w_{2} \neq y$. Then there is $r \in\{1,2\}$ such that $w_{r}^{+} \notin\left\{a_{1}, a_{2}, a_{3}\right\}$. Since $d\left(v, w_{r}^{+}\right)=2$ then $\left|N(v) \cap N\left(w_{r}^{+}\right)\right| \geq 3$ and there exists a vertex $v_{1} \in\left(N(v) \cap N\left(w_{r}^{+}\right)\right) \backslash W_{v}$ together with an $x-y$ path $P_{n+2}^{\Delta}=x \vec{P}_{n}^{\Delta} w_{r} v v_{1} w_{r}^{+} \vec{P}_{n}^{\Delta} y$, a contradiction. So $\left|W_{v}\right| \geq 3$ for each $v \in U_{2}$.

Property 7. Let $v \in U_{2}$. Then $a_{2} \in W_{v}$ for each triangle $a_{1} a_{2} a_{3} a_{1}$ from the set $T\left(P_{n}^{\Delta}\right)$.

Proof. Let $w_{1}, \ldots, w_{p}$ denote vertices of $W_{v}$ occurring on $P_{n}^{\Delta}$ in the order of their indices. By Property $6, p \geq 3$. Suppose $a_{2} \notin W_{v}$ for some triangle $a_{1} a_{2} a_{3} a_{1}$ from the set $T\left(P_{n}^{\Delta}\right)$. Then, by Property $5, a_{1}=w_{k}, a_{3}=w_{k+1}$ and $a_{2}=w_{k}^{+}=w_{k+1}^{-}$for some $w_{k} \in W_{v}$. W.l.o.g. we assume $k<p-1$. (Otherwise we will consider the path $\bar{P}{ }_{n}^{\Delta}$.) Clearly $w_{k+1}^{-} w_{k+1}^{+} \notin E(G)$. Set $Q=W_{v} \backslash\left\{w_{k}, w_{p}\right\}$. Then, by Property 4, we have (3), (4), and (5). Since the vertices $w_{k}$ and $w_{p}$ can be adjacent to each vertex $w_{i}^{+} \in Q^{+}$we have

$$
\sum_{w_{i} \in Q}\left|N(v) \cap N\left(w_{i}^{+}\right)\right| \leq \varepsilon\left(Q, Q^{+}\right)+2(p-2) .
$$

Furthermore,

$$
\sum_{w_{i} \in Q}\left|N\left(w_{i}\right) \backslash\left(N(v) \cup N\left(w_{i}^{+}\right)\right)\right| \geq \varepsilon\left(Q, Q^{+}\right)+p-1
$$

because $w_{k+1}^{-} \notin Q^{+}, w_{k+1}^{-} \in N\left(w_{k+1}\right) \backslash\left(N(v) \cup N\left(w_{k+1}^{+}\right)\right)$and $v \notin Q^{+}, v \in N\left(w_{i}\right) \backslash$ $\left(N\left(w_{i}^{+}\right) \cup N(v)\right)$ for each $w_{i} \in Q$. Clearly, (10) is equivalent to

$$
\sum_{w_{i} \in Q}\left(\left|N\left(w_{i}\right) \backslash\left(N(v) \cup N\left(w_{i}^{+}\right)\right)\right|+1\right) \geq \varepsilon\left(Q, Q^{+}\right)+2(p-2)+1 .
$$

But (9) and (11) together contradict (3).

Property 8. Let $v \in U_{2}$ and $w_{1}, \ldots, w_{p}$ denote vertices of $W_{v}$ occurring on $P_{n}^{\Delta}$ in the order of their indices. Then $w_{i}^{-} w_{i}^{+} \in E(G)$ for each $i=2, \ldots, p-1$. 
Proof. Let $\Delta=a_{1} a_{2} a_{3} a_{1}$ be a triangle from the set $T\left(P_{n}^{\Delta}\right)$. Then, by Property 7, $a_{2}=w_{r}$ for some $r, 1 \leq r \leq p$. W.1.o.g. we assume $r \leq p-1$. (Otherwise we will consider the path $\overleftarrow{P}_{n}^{\Delta}$.) Let us show that

$$
\text { if } k<p-1 \text { and } w_{k}^{-} w_{k}^{+} \in E(G) \text { then } w_{k+1}^{-} w_{k+1}^{+} \in E(G) \text {. }
$$

Set $Q=W_{v} \backslash\left\{w_{k}, w_{p}\right\}$. If $w_{k+1}^{-} w_{k+1}^{+} \notin E(G)$ then, by repeating the arguments in the proof of Property 7, we obtain (3), (4), (5), (9), and (11). But (9) and (11) contradict (3). So, $w_{i}^{-} w_{i}^{+} \in E(G)$ for each $i, r \leq i \leq p-1$. If $r>2$ then we will consider the path $\overleftarrow{P}_{n}^{\Delta}$. Using the above arguments we obtain $w_{i}^{-} w_{i}^{+} \in E(G)$ for each $i, 2 \leq i \leq r-1$.

Now using the above properties we will obtain a contradiction. Let $v \in U_{2}$ and $w_{1}, \ldots, w_{p}$ be vertices of $W_{v}$ occurring on $P_{n}^{\Delta}$ in the order of their indices. By Property $8, w_{i}^{-} w_{i}^{+} \in E(G)$ for each $i=2, \ldots, p-1$. Clearly,

$$
d\left(w_{1}^{+}, v\right)=2, N(v) \cap N\left(w_{1}^{+}\right) \subseteq W_{v} \quad \text { and } \quad \mid N(v) \cap N\left(w_{1}^{+}\right) \geq 3 .
$$

Hence there is a vertex $w_{m} \in W_{v}$ which is adjacent to $w_{1}^{+}$. If $p \geq 4$ then there is an $x-y$ path $\vec{P}_{n+1}^{\Delta_{1}}=x \vec{P}_{n}^{\Delta} w_{1} v w_{m} w_{1}^{+} \vec{P}_{n}^{\Delta} w_{m}^{-} w_{m}^{+} \vec{P}_{n}^{\Delta} y$ with

$$
\Delta_{1}= \begin{cases}w_{2}^{-} w_{2} w_{2}^{+} w_{2}^{-} & \text {if } m>2 \\ w_{3}^{-} w_{3} w_{3}^{+} w_{3}^{-} & \text {if } m=2\end{cases}
$$

a contradiction. So, $p=3$. From (13) we obtain

$$
\left|N(v) \cap N\left(w_{1}^{+}\right)\right|=3 \quad \text { and } \quad w_{1}^{+} w_{i} \in E(G) \quad \text { for } i=1,2,3 .
$$

Since $G$ is connected and $n \leq|V(G)|-2$ there is a vertex $u \in N\left(P_{n}^{\Delta}\right) \backslash\{v\}$. Using Properties 2 and 7 with the vertex $u$ and the triangle $w_{2}^{-} w_{2} w_{2}^{+} w_{2}^{-}$we obtain $w_{2} u \in E(G)$. Clearly, $u v \notin E(G)$. (Otherwise there is an $x-y$ path

$$
P_{n+2}^{\Delta_{1}}=x \vec{P}_{n}^{\Delta} w_{1} v u w_{2} w_{1}^{+} \vec{P}_{n}^{\Delta} w_{2}^{-} w_{2}^{+} \vec{P}_{n}^{\Delta} y
$$

with $\Delta_{1}=v u w_{2} v$, a contradiction.) Furthermore, $w_{1}^{+} u \notin E(G)$. (Otherwise there is an $x-y$ path $P_{n+2}^{\Delta_{1}}=x \vec{P}_{n}^{\Delta} w_{1} v w_{2} u w_{1}^{+} \vec{P}_{n}^{\Delta} w_{2}^{-} w_{2}^{+} \vec{P}_{n}^{\Delta} y$ with $\Delta_{1}=w_{2} u w_{1}^{+} w_{2}$, a contradiction.) So, $w_{2} \in N\left(w_{1}^{+}\right) \cap N(v)$ and $u, v, w_{2}^{+} \in N\left(w_{2}\right) \backslash\left(N(v) \cup N\left(w_{1}^{+}\right)\right)$. Hence, by Proposition 1, we obtain $\left|N(v) \cap N\left(w_{1}^{+}\right)\right| \geq 4$, which contradicts (14). The proof of Theorem 5 is complete.

Theorem 6. Let $G \in L$. Then, for each edge $x y \in E(G)$ and for each integer, $n, 3 \leq n \leq$ $|V(G)|$, (except maybe one $n \in\{3,4\}$ ) there is a cycle of length $n$ containing $x y$.

Proof. Let $x y \in E(G)$. Since $x y$ lies on a triangle or on a quadrangle (see proof of Proposition 3) it is sufficient to prove that there exists an $x-y$ path $P_{n}$ for each $n, 5 \leq n \leq|V(G)|$. By Proposition 3 there exists an $x-y$ path $P_{s}^{\Delta}$ where $4 \leq s \leq 6$. Hence there also exists an $x-y$ path $P_{s-1}$. Suppose there exist an $x-y$ path $P_{i}$ for each $i, s-1 \leq i \leq n-1$, and an $x-y$ path $P_{n}^{\Delta}$, where $s \leq n \leq|V(G)|-1$.

If $n \leq|V(G)|-2$ then, by Theorem 5 , there exists an $x-y$ path $P_{n+t}^{\Delta_{1}}$ where $1 \leq t \leq 2$. If $t=2$ and $\Delta_{1}=w^{-} w w^{+} w^{-}$then we can obtain an $x-y$ path $P_{n+1}$ by deleting the vertex $w$ from $P_{n+2}^{\Delta_{1}}$. 
Suppose now that $n=|V(G)|-1$ and let $v$ be the unique vertex outside $P_{n}^{\Delta}$. Let $w_{1}, \ldots, w_{p}$ be the vertices of $W_{v}$ occurring on $P_{n}^{\Delta}$ in the order of their indices. Since $G$ is 3-connected we have $p \geq 3$. If $w_{i}^{+}=w_{i+1}$ for some $i, 1 \leq i \leq p-1$, then there is a Hamilton $x-y$ path. Let $w_{i}^{+} \neq w_{i+1}$ for each $i=1, \ldots, p-1$. Set $Q=W_{v} \backslash\{y\}$. Clearly (3) holds. Let us show $w_{i}^{+} w_{j}^{+} \in E(G)$ for some $w_{i}, w_{j} \in Q$. Clearly $N(v) \cap N\left(w_{i}^{+}\right) \subseteq W_{v}$ for each $w_{i} \in Q$. If $w_{i}^{+} w_{j}^{+} \notin E(G)$ for each pair of vertices $w_{i}, w_{j} \in Q$ then (6), (7), and (8) hold. But (6) and (8) contradict (3). So $w_{i}^{+} w_{j}^{+} \in E(G)$ for some $w_{i}, w_{j} \in E(G)$ where $i<j$. Then there is a Hamilton $x-y$ path $P_{n+1}=x \vec{P}_{n}^{\Delta} w_{i} v w_{j} \overleftarrow{P}_{n}^{\Delta_{n}} w_{i}^{+} w_{j}^{+} \vec{P}_{n}^{\Delta} y$.

Repetition of our argument shows that there is an $x-y$ path $P_{n}$ for each $n, s \leq n \leq$ $|V(G)|$. This proves the theorem because $4 \leq s \leq 6$.

Using Proposition 2 instead of Proposition 3 and the same arguments as in the proof of Theorem 6 we can prove the following.

Theorem 7. Let $G \in L$ and $x, y$ be two distinct vertices of $G$ with $d(x, y) \geq 2$. Then for each $n, d(x, y)+1 \leq n \leq|V(G)|$, there exists an $x-y$ path $P_{n}$.

Clearly, Theorems 6 and 7 imply Theorem 3. Moreover, from Theorem 6 and Theorem 7 we can obtain the following.

Theorem 8. A graph $G \in L$ is panconnected (and also edge pancyclic) if and only if every edge of $G$ lies in a triangle and a quadrangle.

Corollary 2. A graph $G$ satisfying the condition of Theorem 1 is panconnected if and only if each edge of $G$ lies in a triangle and a quadrangle.

It is not difficult to check that in every graph satisfying the condition of Theorem 2 each edge lies on a triangle and a quadrangle. So, Theorem 2 follows from Corollary 2.

Corollary 3. Let $G$ be a connected $r$-regular graph of order at least 4 where $\mid N(u) \cup$ $N(v) \cup N(w) \mid \leq 2 r-1$ for any path $u w v$ with $u v \notin E(G)$. Then $G$ is panconnected unless $r=2 n$ and $G=\bar{K}_{2 n-1} \vee n K_{2}$ where $n K_{2}$ denote the union of $n$ disjoint copies of $K_{2}$.

Proof. If each edge of $G$ lies in a triangle and a quadrangle then, by Theorem $8, G$ is panconnected. Now suppose that an edge $e=x y$ does not lie in a triangle or a quadrangle. Let $N(x)=\left\{y, v_{1}, \ldots, v_{r-1}\right\}$. If $N(x) \cap N(y)=\emptyset$ then $\left|N(y) \cup N\left(v_{1}\right) \cup N(x)\right| \geq 2 r$ because $G$ is $r$-regular, a contradiction.

So $N(x) \cap N(y) \neq \emptyset$. Without loss of generality we assume that $y v_{1} \in E(G)$. Since $x y$ lies in the triangle $x y v_{1} x$ then, by our assumption, $x y$ does not lie in a quadrangle. Hence $v_{1} v_{i} \notin E(G)$ for each $i=2, \ldots, r-1$. Let $N\left(v_{1}\right)=\left\{x, y, u_{1}, \ldots, u_{r-2}\right\}$. Since $\left|N(x) \cup N\left(v_{i}\right) \cup N\left(v_{1}\right)\right| \leq 2 r-1$ and $\left\{x, y, u_{1}, \ldots, u_{r-2}, v_{1}, \ldots, v_{r-1}\right\} \subseteq N(x) \cup N\left(v_{i}\right) \cup$ $N\left(v_{1}\right)$ then $\left|N(x) \cup N\left(v_{i}\right) \cup N\left(v_{1}\right)\right|=2 r-1$ for each $i=2, \ldots, r-1$. This implies that $N\left(v_{i}\right)=\left\{x, y, u_{1}, \ldots, u_{r-2}\right\}$ for each $i=2, \ldots, r-1$ and $N(y)=\left\{x, v_{1}, \ldots, v_{r-1}\right\}$. If $N\left(u_{j}\right) \backslash\left\{u_{1}, \ldots, u_{r-2}, v_{1}, \ldots, v_{r-1}\right\} \neq \emptyset$ for some $j, 1 \leq j \leq r-2$, then $\mid N\left(u_{j}\right) \cup N\left(v_{1}\right) \cup$ $N(x) \mid \geq 2 r$, a contradiction. So, $N\left(u_{j}\right) \subseteq\left\{u_{1}, \ldots, u_{r-2}, v_{1}, \ldots, v_{r-1}\right\}$ for each $j=$ $1, \ldots, r-2$. Since $G$ is $r$-regular we deduce that $r-2$ is an even number and the subgraph induced by the set $\left\{u_{1}, \ldots, u_{r-2}\right\}$ is a 1-factor. So, $r=2 n$ and $G=\bar{K}_{2 n-1} \vee n K_{2}$.

Let, for each vertex $w$ of a graph $G, M_{2}(w)$ denote the set of vertices $v$ with $d(w, v) \leq 2$.

Corollary 4. Let $G$ be a connected $r$-regular graph of order at least 4 where $\left|M_{2}(w)\right| \leq$ $2 r-1$ for each $w \in V(G)$. Then $G$ is panconnected unless $r=2 n$ and $G=\bar{K}_{2 n-1} \vee n K_{2}$. 
Proof. Let $u w v$ be a path of $G$ with $u v \notin E(G)$. Clearly, $N(u) \cup N(v) \cup N(w) \subseteq M_{2}(w)$. Hence, $\left|M_{2}(w)\right| \leq 2 r-1$ implies $|N(u) \cup N(v) \cup N(w)| \leq 2 r-1$. Therefore, by Corollary $3, G$ is panconnected.

\section{References}

[1] A. S. Asratian, O. A. Ambartsumian, and G. V. Sarkisian, Some local conditions for the hamiltonicity and pancyclicity of a graph, Docl. Acad. Nauk Armenian SSR 91 (1990), 19-22 (Russian).

[2] A. S. Asratian and G. V. Sarkisian, Some panconnected and pancyclic properties of graphs with a local Ore-type condition, Graphs and Combinat., in press.

[3] A. S. Asratian and G. V. Sarkisian, On cyclic properties of some hamiltonian graphs, Diskretnaja Matemat. 3 (1991), 91-104. (Russian.)

[4] K. A. Berman, Proof of a conjecture of Häggkvist on cycles and independent edges, Discrete Math. 46 (1983), 9-13.

[5] J. A. Bondy, Pancyclic graphs I, J. Combin. Theory B 11 (1971), 80-84.

[6] J. A. Bondy and U. S. R. Murty, Graph Theory with Applications, MacMillan, London and Elsevier, New York (1976).

[7] Cai Xiao-tao, A short proof for the Faudree-Schelp theorem on path connected graphs, J. Graph Theory 8 (1984), 109-110.

[8] R. J. Faudree and R. H. Schelp, Path connected graphs, Acta Math. Acad. Sci. Hungar. 25 (1974), 313-319.

[9] R. Häggkvist, On F-Hamiltonian graphs, in: (eds. J. A. Bondy and U. S. R. Murty, Graph Theory and Related Topics, Academic Press, New York (1979), 219-231.

[10] R. Häggkvist, R. J. Faudree, and R. H. Schelp, Pancyclic graphs-connected Ramsey number, Ars Combinatoria 11 (1981), 37-49.

[11] A. S. Hasratian and N. K. Khachatrian, Some localization theorems on hamiltonian circuits, $J$. Combin. Theory B 49 (1990), 287-294.

[12] O. Ore, Hamilton connected graphs, J. Math. Pures Appl. 42 (1963), 21-27.

[13] J. E. Williamson, Panconnected graphs II, Period. Math. Hungar. 8 (1977), 105-116.

[14] Zhang Ke Min and D. A. Holton, On edge-pancyclic graphs, Soochow J. Math. 19 (1) (1993), $37-41$.

[15] Zhang Ke Min, D. A. Holton, and Sheng Bau, On generalized vertex-pancyclic graphs, Chinese J. Math. 21 (1) (1993), 91-98.

Received August 2, 1994 